\title{
Trickle and single infection with Discocotyle sagittata (Monogenea: Polyopisthocotylea): effect of exposure mode on parasite abundance and development
}

\author{
Miguel Rubio-Godoy and Richard C. Tinsley
}

School of Biological Sciences, University of Bristol, Woodland Rd., Bristol BS8 1UG, UK

Key words: Monogenea, Discocotyle sagittata, Oncorhynchus mykiss, trickle infection, single infection, competition, migration

\begin{abstract}
Experimental infection of rainbow trout Oncorhynchus mykiss (Walbaum) with the monogenean Discocotyle sagittata (Leuckart, 1842) allowed comparison between trickle and single exposure, two infection modes demonstrated to occur in the wild. Both types of infection resulted in mean larval attachment success around $50 \%$, which was significantly dependent on dose of infective larvae used $(P<0.0001)$, but was not affected by mode of infection $(P=0.244)$. Worms recovered from fish exposed to the same number of oncomiracidia but different mode of infection differed in their rate of development. The developmental stage attained by parasites was significantly affected by number of infective larvae used $(P=0.005)$, and by the interaction between dose and mode of infection $(P=0.026)$, suggesting competition among attached larvae. Statistical analysis demonstrated that in the early stages of infestation, worm distribution over the gill arches can be explained by the relative amount of water flowing over them. One, two and three months post-infection parasite numbers were comparable $(P=0.805)$, but their observed distribution gradually decreased in gill arches III and IV and increased in gill arch I, suggesting that parasites migrate after initial attachment. These results reproduce phenomena observed in the field, indicating that the experimental infection system could be employed to study infection dynamics and host-parasite interactions under controlled conditions.
\end{abstract}

Discocotyle sagittata (Leuckart, 1842) Diesing, 1850 is a sanguinivorous polyopisthocotylean monogenean occurring on the gills of freshwater salmonid fishes, with an extensive geographical distribution in the Northern Hemisphere. It occurs at low intensities in the wild (Paling 1965, Valtonen et al. 1990); however, heavy infections by $D$. sagittata can result in deleterious effects on the host, especially in the context of fish farming (Roberts 1978, Thoney and Hargis 1991, Gannicott 1997). In recent years, rainbow trout Oncorhynchus mykiss (Walbaum) reared in certain farms in the Isle of Man have been shown to harbour high parasite burdens (>1000/fish) and exhibit high mortality rates (Gannicott 1997) due to parasite-induced anaemia.

Temperature is the single most important abiotic factor affecting $D$. sagittata: egg production, development and viability are highest within the range $13-18^{\circ} \mathrm{C}$ in the UK and decline at temperatures both below and above this (Gannicott and Tinsley 1998b); oncomiracidia exhibit maximal survival at $6^{\circ} \mathrm{C}$, declining with increasing temperature (Gannicott and Tinsley 1998a); and parasite developmental rate is highest between $13-18^{\circ} \mathrm{C}$ (Gannicott 1997). Epidemiological studies in farms over several years indicate this applies to natural conditions (Gannicott 1997): no parasite transmission occurs during the cold part of the year (end November - early May), while it is continuous with water temperatures $>10^{\circ} \mathrm{C}$ (end May - early November). It is thus reasonable to propose that both trickle and single infection episodes can occur naturally: the first throughout the warm part of the year when parasite egg laying, embryo development and hatching of infective larvae occur without interruption; the latter when viable overwintered eggs hatch en masse in early summer. Since these two infection scenarios occur at different temperatures, fish immunocompetence may differ between them (Tatner 1996).

The effect of trickle and single infection on parasite population dynamics and on the host immune response has been studied in a variety of systems. For instance, sheep have been experimentally exposed to single and trickle infections with the nematodes Haemonchus contortus (Barger et al. 1985, McClure et al. 1998), Trichostrongylus colubriformis (Dobson et al. 1990, McClure et al. 1998), and Teladorsagia circumcincta (Gruner et al. 1994); pigs to the nematode Oesophagostomum dentatum (Barnes 1997, Talvik et al. 1999) or Taenia asiatica (Fall et al. 1996); and calves to Cooperia oncophora (Van Diemen et al. 1996), Mecistocirrus digitatus (Van Aken et al. 1997) and Fasciola hepatica (Bossaert et al. 2000a, b). A common feature of these studies is that parasite burdens reached by either single or trickle infection are comparable. A second common finding is that higher doses generally result in reduced parasite development and/or fecundity. 
Considering the effects of temperature on both host and parasite, rainbow trout (O. mykiss) were exposed to experimental trickle and single $D$. sagittata infection at $13^{\circ} \mathrm{C}$, a controlled, permissive temperature. Thus, the experiments we report aimed at determining the effect of exposure mode on parasite establishment success, development and distribution.

\section{MATERIALS AND METHODS}

Naturally-infected fish. Naturally-infected rainbow trout (Oncorhynchus mykiss) were obtained from two hatcheries in the Isle of Man. Fish were killed and their gill arches dissected and preserved in $10 \%$ formalin for later microscopic analysis.

Fish for experimental procedures. Oncorhynchus mykiss fingerlings (ca $20 \mathrm{~cm}$ long) were purchased from a parasitefree hatchery. Batches of 30-50 fish were maintained in 500litre tanks in the University of Bristol, kept at $13^{\circ} \mathrm{C}$ and fed $3.0 \mathrm{~mm}$ commercial feed pellets (Trout floating 30, BOCM Pauls, UK). Animals were allowed at least 7 days to recover from transport-related stress before the infection procedures.

Parasites. Rainbow trout naturally-infected with Discocotyle sagittata from the Isle of Man were transported to Bristol and housed at $13^{\circ} \mathrm{C}$ under a light:dark regimen of 12:12 h. Parasite eggs were collected by filtration through a $125 \mu \mathrm{m}$ nylon mesh sieve; the filtrate was washed twice with dechlorinated water and decanted successively to obtain a residual volume of ca $25 \mathrm{ml}$ containing parasite eggs. This was transferred to a crystallising dish and rotated gently so that eggs accumulated in its centre. Under a dissecting microscope, these were transferred using a Pasteur pipette in batches of approximately 100 to clean $3.5 \mathrm{~cm}$ diameter Petri dishes and kept at $13^{\circ} \mathrm{C}$ under $12 \mathrm{~L}: 12 \mathrm{D}$ photoperiod. Under these con-ditions, eggs exhibit high viability and hatch within ca 28 days (Gannicott 1997, Gannicott and Tinsley 1997). After em-bryonation, egg batches were placed in an incubator at $9^{\circ} \mathrm{C}$ and left in total darkness for $1 \mathrm{~h}$; this enabled mass collection of recently-emerged infective stages since $94 \%$ of eggs hatch within the first hour after the onset of darkness (Gannicott and Tinsley 1997). Dishes were screened under a dissecting microscope after the $1 \mathrm{~h}$ hatching period, and actively-swimming oncomiracidia were collected with a Pasteur pipette and placed in batches of 50 in Petri dishes. The larvae were microscopically checked for viability (and accidentally transferred eggs) and were never allowed to age $>2 \mathrm{~h}$ before use.

Infection procedure. Fish were placed individually in infection tanks (ca $11.51,13^{\circ} \mathrm{C}$ ) and exposed to controlled numbers of active oncomiracidia for $24 \mathrm{~h}$, the first $12 \mathrm{~h}$ of which were in darkness. At $13^{\circ} \mathrm{C}$ D. sagittata oncomiracidia exhibit $50 \%$ survival after $12 \mathrm{~h}$, and $25 \%$ after $24 \mathrm{~h}$ (Gannicott and Tinsley 1998a). A preliminary experiment (data not shown) indicated all fish thus exposed to oncomiracidia became infected in a dose-dependent manner (one-way ANOVA; $\left.F_{1}=53.533, P<0.0001\right)$. To standardise stressrelated and environmental effects, all fish groups were kept in identical tanks and exposed to similar handling.

All experimental infection procedures were carried out with fish from the same batch. Each experimental group consisted of 8-10 fish which were exposed individually to parasites by trickle or single infection. Table 1 shows the number of oncomiracidia used and the week when experimental groups were exposed to infection. As a control to the effects of handling-related stress, experimental groups that had been exposed to a single infection were sham-infected (Table 1, S; i.e., placed in an infection tank containing no active larvae at the time intervals shown). Fish in both trickle and single infection experiments were dissected 8 weeks after initial exposure.

A third experimental set was exposed to 100 oncomiracidia once, kept at $13^{\circ} \mathrm{C}$ and dissected 1,2 or 3 months postinfection (p.i.). No re-infection was possible during this maintenance period.

Table 1. Discocotyle sagittata trickle and single infection schedule. Experimental groups $(\mathrm{n}=8-10)$ of rainbow trout were exposed to the numbers of oncomiracidia shown or sham-infected (S) as controls at the time intervals shown.

\begin{tabular}{|l|c|c|c|c|c|}
\hline & & \multicolumn{4}{|c|}{ Week } \\
& Group & 1 & 2 & 3 & 4 \\
\hline \multirow{4}{*}{ Trickle } & $50 \times 1$ & 50 & & & \\
exposure & $50 \times 2$ & 50 & 50 & & \\
& $50 \times 3$ & 50 & 50 & 50 & \\
& $50 \times 4$ & 50 & 50 & 50 & 50 \\
\hline Single & $100 \times 1$ & 100 & $\mathrm{~S}$ & & \\
exposure & $200 \times 1$ & 200 & $\mathrm{~S}$ & $\mathrm{~S}$ & $\mathrm{~S}$ \\
\hline
\end{tabular}

Parasite counts. Experimentally infected fish were anaesthetised terminally with $0.05 \%$ MS222, bled by caudal vein puncture, weighed (after exsanguination) and their fork length was measured to $0.1 \mathrm{~cm}$ accuracy. Gills were removed and placed in a Petri dish and individual gill arches separated further immediately afterwards. These were examined under the dissecting microscope and the number of parasites per gill arch was recorded, noting whether worms were located dorsally or ventrally. Individual gill arches were numbered from I for the most anterior to IV for the most posterior and smallest arch; detached worms were included in the total worm counts and developmental stage calculations, but not in the positional analysis. The same procedure was used to inspect the preserved gill samples from the Isle of Man.

Parasites were grouped in different (categorical) developmental cohorts based on the number of pairs of clamps they had grown, as previously described by Gannicott and Tinsley (1997, 1998a, b): freshly hatched parasites possess one pair of clamps (developmental cohort 1 ) and $2^{\text {nd }}, 3^{\text {rd }}$ and $4^{\text {th }}$ pairs develop in succession; cohort 4 includes worms with 4 pairs of clamps but not yet reproducing; "adults" are sexually mature and actively producing eggs and were assigned an arbitrary value of 4.5 for the purpose of a linear graphical representation, not implying the parasites had actually developed more than 4 pairs of clamps. Intermediate values were assigned when parasites showed noticeably smaller clamps than the next cohort.

Age cohort data were transformed into continuous variables by calculating the mean number of pairs of clamps attained by worms recovered from individual fish (including unattached worms). This average was called the Developmental Index and was calculated as follows: 
$\begin{aligned} & \text { Developmental } \\ & \text { Index }\end{aligned}=\frac{\sum_{4.5}^{1} \text { (worms recovered } \times \text { developmental cohort) }}{\text { Total number of worms }}$

The Developmental Index thus ranges between the values of 1 for populations containing only freshly attached worms, and 4.5 for populations composed entirely of sexually mature parasites.

Statistical analysis. Data were analysed with the statistical package SPSS for Windows 10.0. Pooled results from other experiments $(n=73)$ indicate parasite burdens obtained one month post experimental infection were distributed normally (data not shown; One-sample Kolmogorov-Smirnov Z = 0.512, Asympt. Sign. (2-tailed) $=0.956)$. One-way ANOVA was used to analyse the effect of dose (number of oncomiracidia) on numbers of worms recovered in each treatment group, and on the position of worms on the gill arches (plus a Tukey test to detect significant differences between groups); the significance level was set at $P<0.05$. A best-fit regression was calculated for the number of worms established in relation to the number of active larvae employed in the experimental exposure. Univariate ANOVA (General Linear Model analysis) was used to compare the numbers of worms recovered and the Developmental Indices obtained in the experimental trickle and single infections, as well as the distribution of worms 1, 2 and 3 months p.i.; in all instances, fish length and weight were included in the analysis as covariates, and residual distribution was tested for normality. A Chi-squared goodness of fit analysis was employed to assess the relationship of the number of worms attached to each gill arch and the relative amounts of water flowing over each (Paling 1968); the null hypothesis was that the distribution of parasites over the gill arches is positively related to the estimated amount of water flowing over each arch. Predicted numbers of parasites per gill arch were obtained by multiplying the number of worms actually recovered per fish by a factor unique to each arch based on the percentage of the total respiratory flow it receives (arch $\mathrm{I}=$ 0.242 ; $\operatorname{arch}$ II $=0.300$; $\operatorname{arch}$ III $=0.282$; $\operatorname{arch}$ IV $=0.176$; Paling 1968)

\section{RESULTS}

\section{Natural trickle and single infections}

Naturally-infected rainbow trout from the Isle of Man hatcheries studied provide evidence of both trickle and single infection. Fig. 1 shows that fish collected in late autumn (end November) harbour parasites belonging to all developmental stages, reflecting gradual (trickle) infection occurring throughout the summer. Fig. 2 illustrates the simultaneous presence of high numbers of both freshly attached and adult worms in a sample taken in early summer (late May); the large number of synchronous 1-pair of clamps parasites resulted from mass (single) invasion occurring over a limited period (1-2 weeks)

Experimental trickle and single infections
All fish infected experimentally harboured parasites at the time of dissection. Experimental trickle infection resulted in an attachment efficiency showing significant variation (Table 2 and Fig. 3). This was particularly noticeable in the group exposed to a total of 200 infective larvae, exhibiting infection success rates ranging from 41 to $77 \%$. However, experimental trickle infection displayed a significant dose-dependent relationship (one-way ANOVA; $\mathrm{F}_{1}=98.284, \mathrm{P}<0.0001$; linear regression equation $\mathrm{y}=0.489$ oncomiracidia + $\left.9.421 ; \mathrm{R}^{2}=0.743\right)$. Likewise, fish exposed to a single infection presented variable infection success (Table 2 and Fig. 4), but the effect of infective dose was nonetheless significant (one-way ANOVA; $\mathrm{F}_{1}=82.519$, $\mathrm{P}<0.0001$; linear regression equation $\mathrm{y}=0.375$ oncomiracidia $\left.+15.66 ; \mathrm{R}^{2}=0.767\right)$.

All fish groups were comparable in terms of length (mean $\pm \mathrm{SE} ; 19.5 \pm 0.18 \mathrm{~cm})$ and weight $(89.2 \pm 2.65$ g). Neither fish length nor weight had a significant effect on the number of worms recovered $\left(\mathrm{F}_{1}\right.$ Length $=$ $1.44, \mathrm{P}=0.236 ; \mathrm{F}_{1}$ Weight $\left.=0.749, \mathrm{P}=0.391\right)$. Overall, mode of infection had a non significant effect on worm recovery $\left(\mathrm{F}_{1}=11.391, \mathrm{P}=0.244\right)$, but the effect of infective dose was highly significant $\left(\mathrm{F}_{3}=27.703, \mathrm{P}<\right.$ 0.0001).

\section{Parasite developmental stage}

Fig. 5 shows the observed frequencies of parasite developmental stages found 8 weeks after initial infection; this corresponds to 8 weeks post-infection (p.i.) for single, and 4-8 weeks p.i. for trickle infections. Developmental Indices (DI) attained in fish exposed by either trickle or single infection were calculated (Table 2). Parasite development in fish exposed to 100 oncomiracidia by different mode of exposure was similar (Table 2 and Fig. 5). In fish exposed to a total of 200 oncomiracidia all worms recovered in the single exposure group were the same age as the oldest cohort in the trickle-infected fish, considering all infestations had been of equal duration. However, parasites were less developed in the single exposure group (Fig. 5), as confirmed by the DI (Table 2). Univariate ANOVA showed experimental group (trickle or single) had no significant effect on the DI $\left(\mathrm{F}_{1}=0.049, \mathrm{P}=0.826\right)$, whereas both infective dose (100 and 200 larvae; $F_{1}=$ $9.253, \mathrm{P}=0.005)$ and the interaction between experimental group and infective dose $\left(\mathrm{F}_{1}=5.396, \mathrm{P}=0.026\right)$ significantly affected the DI attained. This would suggest that parasite development is most affected during high dose single infections.

\section{Parasite distribution on the gills}

Parasites showed no lateral preference for attachment (right $v s$ left gill arches). In all treatment groups, more worms were found on the ventral than on the dorsal area of the gills (Table 2), but this difference was not significant (one-way ANOVA; $\mathrm{F}_{5}=0.364, \mathrm{P}=0.871$ ). 
In trickle and single infection groups, worm distribution nver the gill arrhes $(8$ vxeele after initial

exposure) can be explained by the relative amount of

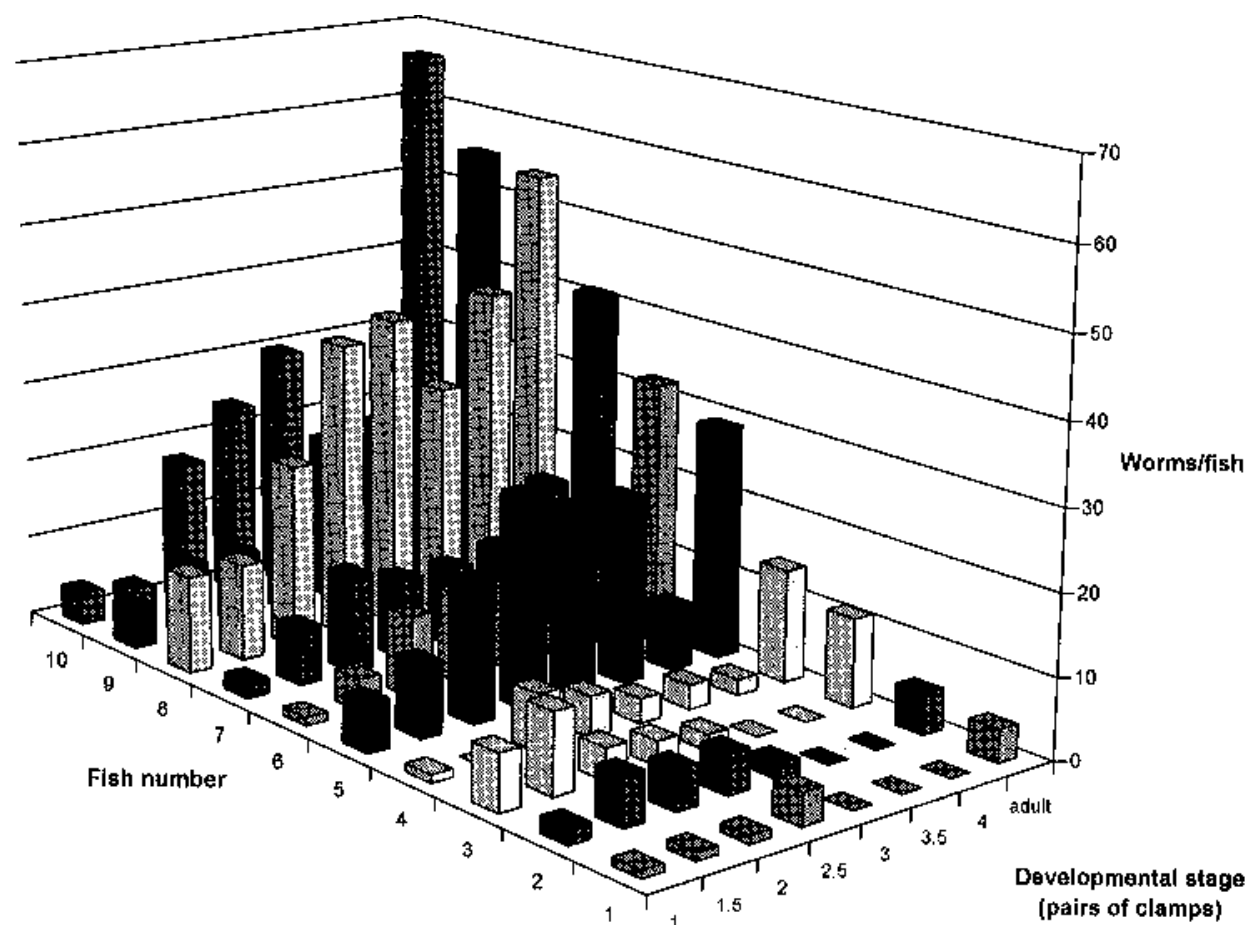

Fig. 1. Developmental stage distribution of Discocotyle sagittata on the gills of individual two-year-old rainbow trout $(\mathrm{n}=10)$, sampled in the Isle of Man at the end of autumn 2000.

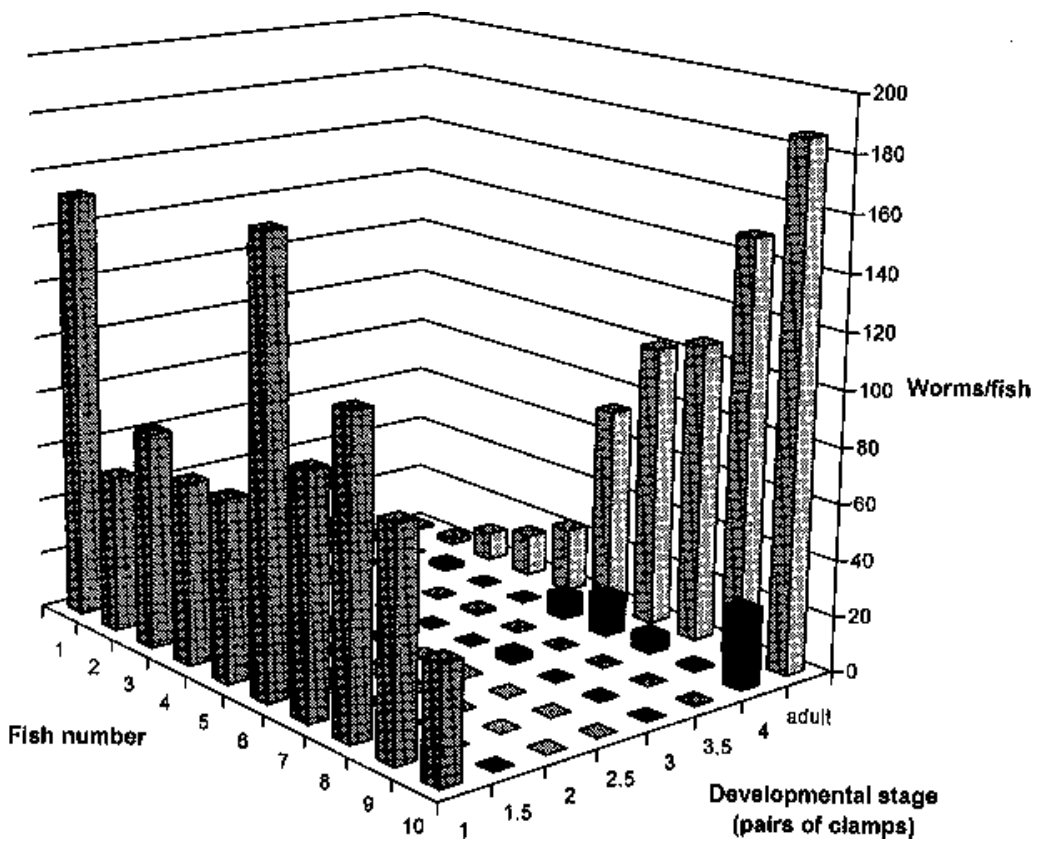

Fig. 2. Developmental stage distribution of Discocotyle sagittata on the gills of individual two-year-old rainbow trout $(\mathrm{n}=10)$, sampled in the Isle of Man at the beginning of summer 2000. 


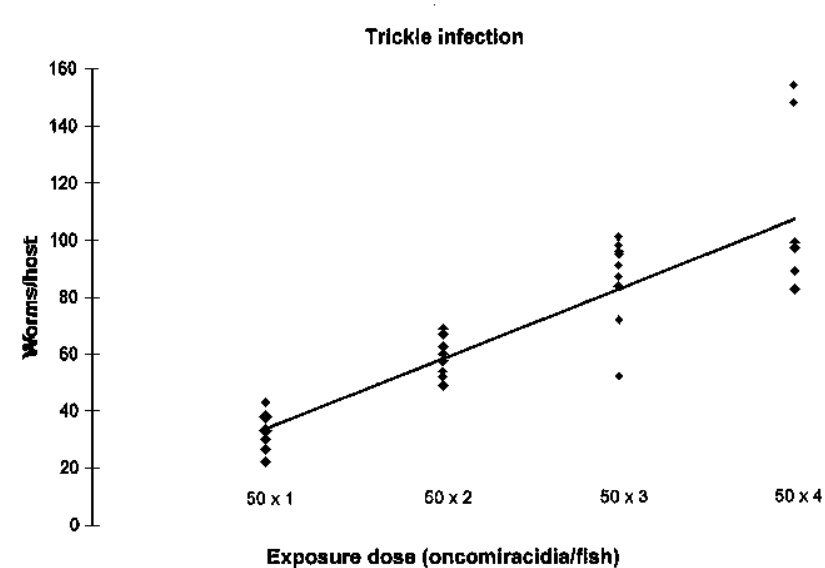

Fig. 3. Experimental trickle infection with Discocotyle sagittata. Fish were exposed to 50 oncomiracidia once $(50 \times 1)$, twice $(50 \times 2)$, three $(50 \times 3)$ or four $(50 \times 4)$ times at oneweek intervals and dissected 8 weeks post-primary infection.
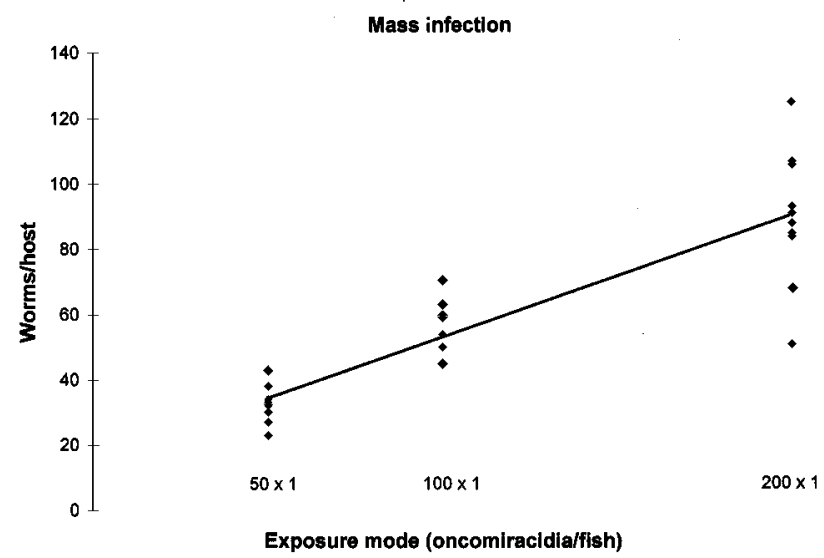

Fig. 4. Experimental single infection with Discocotyle sagittata. Fish were exposed once to 50, 100 or 200 oncomiracidia and then sham-infected once $(100 \times 1)$ or three times $(200 \times 1)$ at weekly intervals.

Table 2. Number and position of worms recovered from gills 8 weeks after experimental trickle or single infection with Discocotyle sagittata oncomiracidia.

\begin{tabular}{|c|c|c|c|c|c|c|}
\hline \multirow{2}{*}{ Treatment } & \multirow{2}{*}{$\mathrm{n}$} & \multirow{2}{*}{$\begin{array}{c}\text { Worms recovered } \\
\text { Mean } \pm \mathrm{SE}\end{array}$} & \multirow{2}{*}{$\begin{array}{c}\text { Infection success } \\
\%\end{array}$} & \multicolumn{2}{|c|}{ Worm position $\% \pm \mathrm{SE}$} & \multirow{2}{*}{$\begin{array}{c}\text { Developmental } \\
\text { Index } \pm \mathrm{SE}\end{array}$} \\
\hline & & & & Dorsal & Ventral & \\
\hline $50 \times 1$ & 9 & $32.4 \pm 1.94$ & 64.9 & $42.4 \pm 3.29$ & $57.6 \pm 3.29$ & $2.11 \pm 0.16$ \\
\hline $50 \times 2$ & 10 & $58.6 \pm 2.01$ & 58.6 & $45.2 \pm 3.28$ & $54.8 \pm 3.28$ & $2.27 \pm 0.10$ \\
\hline $50 \times 3$ & 9 & $86.2 \pm 5.18$ & 57.5 & $46.8 \pm 2.56$ & $53.2 \pm 2.56$ & $2.35 \pm 0.13$ \\
\hline $50 \times 4$ & 8 & $104.4 \pm 10.44$ & 52.2 & $44.8 \pm 7.07$ & $55.2 \pm 7.07$ & $2.16 \pm 0.24$ \\
\hline $100 \times 1$ & 9 & $64.1 \pm 8.05$ & 60.6 & $39.7 \pm 3.03$ & $60.3 \pm 3.03$ & $2.65 \pm 0.08$ \\
\hline $200 \times 1$ & 10 & $85.9 \pm 5.86$ & 44.9 & $43.1 \pm 4.59$ & $56.9 \pm 4.59$ & $1.84 \pm 0.16$ \\
\hline
\end{tabular}

Table 3. Distribution (frequency) and mean Developmental Index (DI) of worms on different gill arches for experimental and natural infections with Discocotyle sagittata.

\begin{tabular}{|c|c|c|c|c|}
\hline & Gill arch I & Gill arch II & Gill arch III & Gill arch IV \\
\hline \multicolumn{5}{|c|}{ Experimental (laboratory) single infection } \\
\hline 1 mo p.i. & 0.16 & 0.35 & 0.31 & 0.18 \\
DI \pm SE & $1.92 \pm 0.11$ & $1.84 \pm 0.16$ & $1.88 \pm 0.11$ & $1.91 \pm 0.11$ \\
2 mo p.i. & 0.26 & 0.32 & 0.27 & 0.15 \\
DI \pm SE & $3.10 \pm 0.17$ & $2.92 \pm 0.10$ & $2.82 \pm 0.10$ & $2.87 \pm 0.10$ \\
3 mo p.i. & 0.31 & 0.35 & 0.22 & 0.12 \\
DI \pm SE & $4.46 \pm 0.03$ & $4.43 \pm 0.05$ & $4.36 \pm 0.05$ & $4.40 \pm 0.05$ \\
\hline \multicolumn{5}{|c|}{ Natural (field) mass infection } \\
\hline Juvenile & 0.09 & 0.07 & 0.17 & 0.22 \\
Mature & 0.13 & 0.15 & 0.10 & 0.07 \\
DI \pm SE & $2.53 \pm 0.34$ & $2.81 \pm 0.37$ & $2.14 \pm 0.29$ & $1.73 \pm 0.24$ \\
\hline
\end{tabular}

Note: Experimental groups $n=9$; natural groups $n=10$. Experimentally-infected fish were exposed to 100 oncomiracidia once and dissected 1, 2 or 3 months post infection (mo p.i.). In the natural mass infection group, "Juvenile" refers to worms with 1 to 3.5 pairs of clamps, and "Mature" to those bearing 4 pairs of clamps / having reached sexual maturity. 


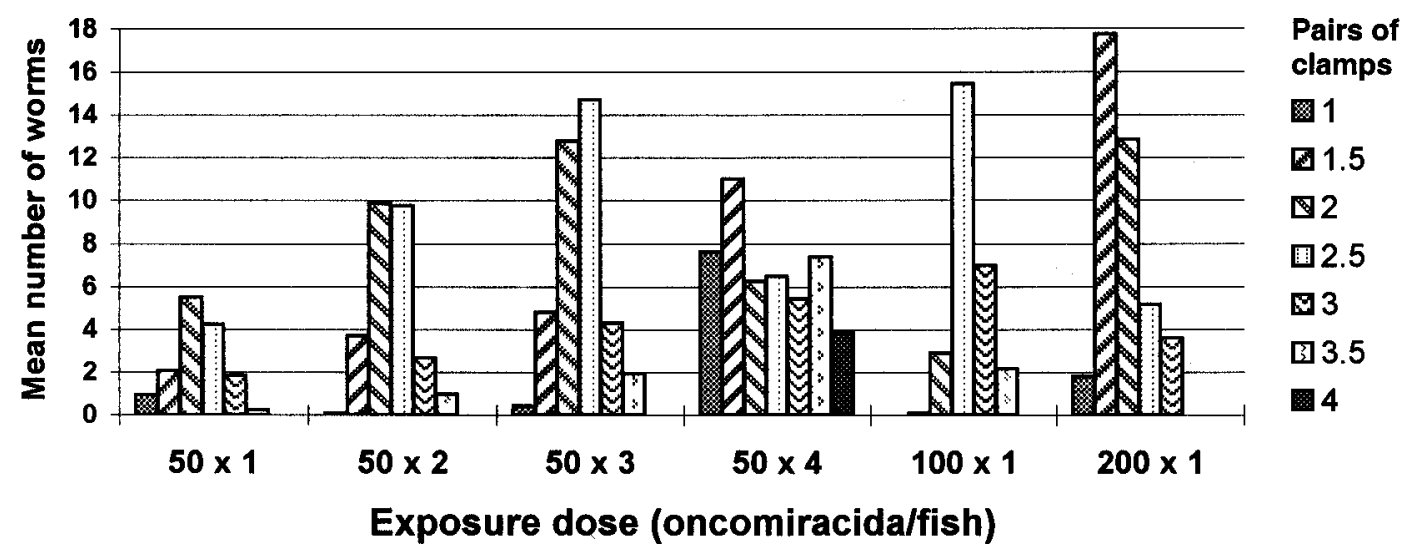

Fig. 5. Mean number of worms recovered per developmental stage for trickle- $(50 \times 1,50 \times 2,50 \times 3$ and $50 \times 4)$ and singleinfected $(100 \times 1$ and $200 \times 1)$ fish. Duration of infection was 4-8 weeks for trickle- and 8 weeks for single-infected groups. Developmental stage was assessed by the number of pairs of clamps grown by the parasites.

water flowing over them (Fig. 6). The central gill arches (II and III) receive most of the respiratory current (Paling 1968) and most parasites were found there (Fig. $6)$; thus, predicted and observed distributions did not differ significantly in any experimental group.

Fish infected with 100 oncomiracidia once were dissected 1, 2 and 3 months p.i. and the mean number of worms $( \pm \mathrm{SE})$ recovered in the 3 groups ( 1 mo p.i. 57.6 \pm 4.35 worms; 2 mo p.i. $53 \pm 6.2$ worms; 3 mo p.i. 52.3 \pm 3.40 worms) was not significantly different (Univariate ANOVA; $\left.\mathrm{F}_{2}=0.219, \mathrm{P}=0.805\right)$. However, the distribution of worms on the gill arches varied significantly 1, 2 and 3 mo p.i. (Table 3; Univariate ANOVA; $\left.\mathrm{F}_{3}=12.237, \mathrm{P}<0.0001\right)$. As would be expected, the mean developmental stage attained differed significantly between the 3 groups (Table 3; Univariate ANOVA; $\left.F_{1}=145.368, P<0.0001\right)$. In naturally mass-infected fish (Fig. 2), freshly attached worms occurred mainly on gill arches III and IV, while older and adult parasites were primarily found on arches I and II (Table 3).

\section{DISCUSSION}

\section{Natural trickle and single infections}

We present evidence for the occurrence of trickle and mass infection by Discocotyle sagittata under the conditions found in fish hatcheries. At the beginning of the transmission cycle in early summer (late May), 2-yearold fish harboured two distinct parasite populations: adult worms acquired during the previous summer and large numbers of recently attached juveniles with 1 pair of clamps, reflecting an age of 1-2 weeks p.i. (Gannicott 1997). Thus, burdens of these juveniles (mean 89 worms/fish, range 41-160) resulted from a recent massive invasion. This single infection might be considered the first of a series of trickle infections; however, more extensive seasonal studies (not presented here, but see Gannicott 1997) show that this pulse of early summer invasion is distinctive, attributable to the mass hatching of eggs that have accumulated on pond bottoms during the preceding few months. At the end of autumn (late November), $2+$ fish carried mostly older or sexuallymature worms, along with a series of juveniles exhibiting a wide range of developing stages including freshly attached parasites. Temperature may be the main abiotic factor regulating the observed parasite population dynamics: under controlled conditions in the laboratory, adult parasites (at burdens of 1-17 worms/fish) constantly release eggs at a rate that varies from a mean daily per capita output of 6.8 at $13^{\circ} \mathrm{C}$ to 12.2 at $18^{\circ} \mathrm{C}$ (Gannicott and Tinsley 1998b); exposed to summer mean water temperatures $\left(13-15^{\circ} \mathrm{C}\right)$, eggs hatch after short incubation periods (20-28 days) and exhibit high viability (91-95\%; Gannicott and Tinsley 1998b). In contrast, eggs develop very slowly during winter and exhibit low viability, but a proportion hatch when returned to favourable temperatures (Gannicott and Tinsley 1997). A similar pattern of permissive and inhibitory temperature-related effects has been demonstrated for larval survival and behaviour of $D$. sagittata (Gannicott and Tinsley 1997). As shown in Table 3, juvenile worms were mainly found on gill arches III and IV, while adult parasites preferentially occupied the first two arches, as reported previously (Gannicott 1997). Presence of adult parasites on gill arch II may have inhibited oncomiracidial attachment during natural mass infection, since both gill arches II and III were frequently occupied by juvenile worms after experimental exposure (Table 3). However, the same distribution trend for juvenile parasites was found in both fish with few or no adult worms (e.g., fish 1-5 in Fig. 2) and those with considerable numbers of mature parasites (fish 6-10). 


\section{Trickle infection}
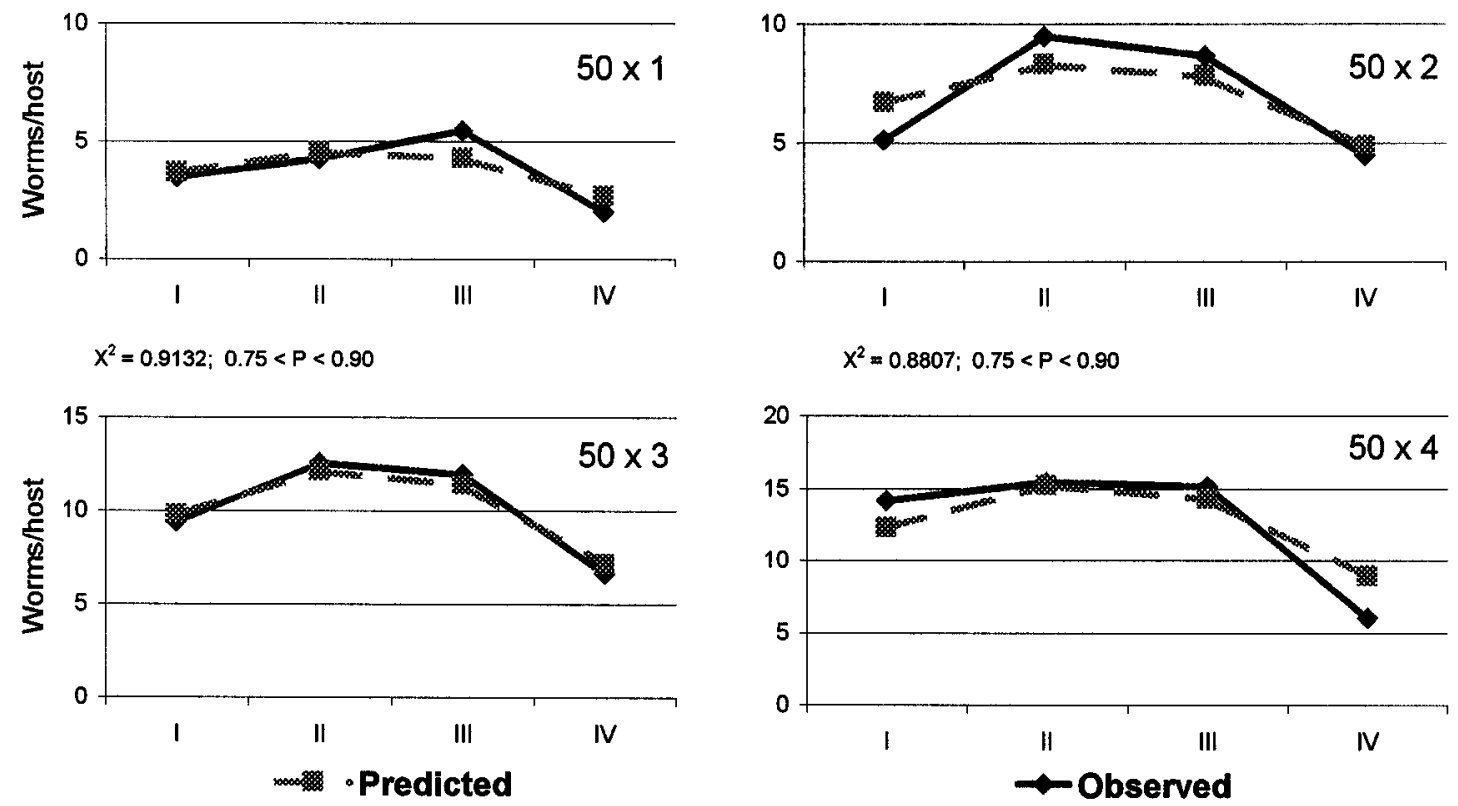

$X^{2}=0.9927 ; 0.75<P<0.90$

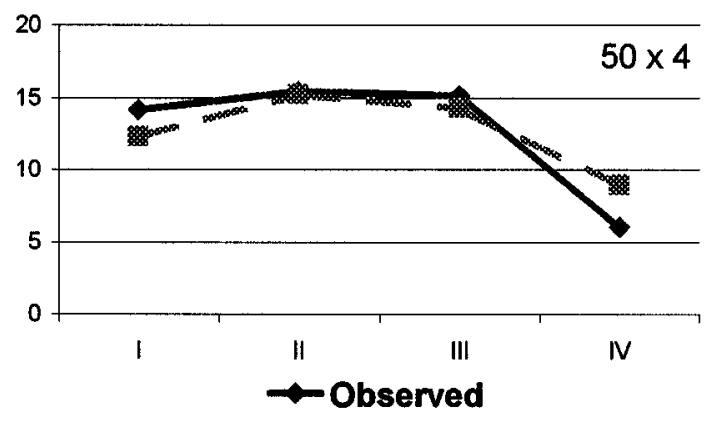

$X^{2}=0.7315 ; 0.75<P<0.90$

Single infection

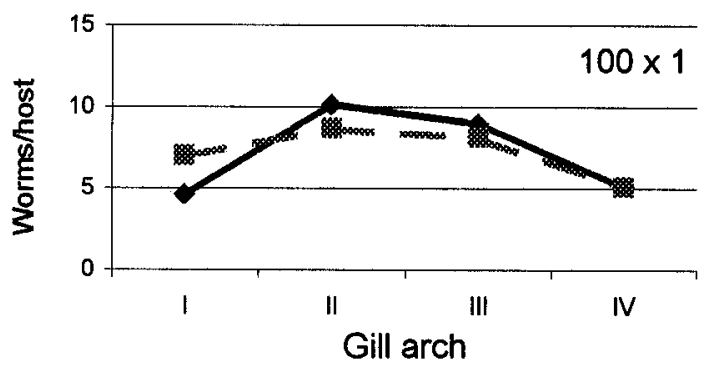

$X^{2}=0.7691 ; 0.75<P<0.90$

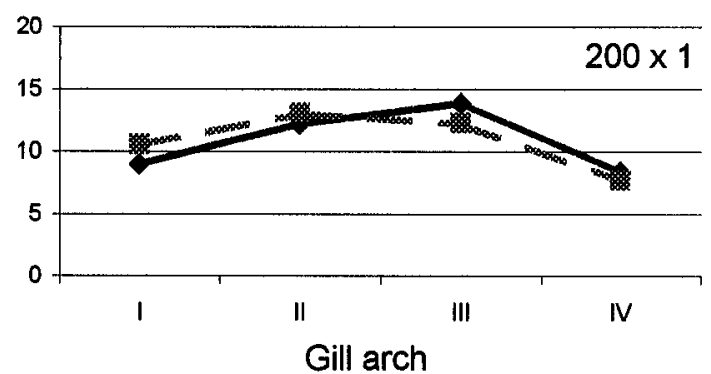

$X^{2}=0.9034 ; 0.75<P<0.90$

Fig. 6. Chi-squared goodness of fit analysis for predicted and observed Discocotyle sagittata distributions over gill arches per experimental group. Chi-squared and $\mathrm{P}$ values are shown for individual experimental groups. Null hypothesis: Parasite distribution on gill arches relates positively to estimated water flow rates over them; Alternative hypothesis: Parasite distribution on gill arches is independent of estimated water flow rates. Notes on the figure legends: Observed $=$ actual mean number of worms recovered/gill arch for every experimental group; Predicted $=($ total number of worms recovered $) \times($ estimated water flow rate per gill arch based on Paling 1968).

\section{Experimental trickle and single infections}

Trickle and single infection resulted in comparable parasite burdens 8 weeks p.i. which exhibited a clear dose-dependent relationship. Likewise, similar parasite burdens were found after single and trickle infections with Teladorsagia circumcincta (Gruner et al. 1994), Taenia asiatica (Fall et al. 1996), Mecistocirrus digitatus (Van Aken et al. 1997), Oesophagostomum dentatum (Barnes 1997) and Fasciola hepatica (Bossaert et al. 2000a, b). However, despite similar burdens, mode of infection has been found to have an

effect on parasite development and/or fecundity, which is suggestive of competition and/or immune effects. For instance, lower $O$. dentatum burdens resulted in larger female worms and higher egg output (Barnes 1997), probably related to developmental delay in higher infection doses. Moreover, the adverse effects of higher infection doses have been shown to act upon the next parasite generation, as illustrated by decreased infectivity of $O$. dentatum larvae derived from eggs 
excreted in high dose trickle-infected pigs (Talvik et al. 1999).

\section{Parasite developmental stage}

Burdens of $D$. sagittata resulting from single or trickle infection were not significantly different, but the mean developmental stage attained by single exposure parasites was significantly lower. Thus, the number of oncomiracidia establishing simultaneously may lead to competitive interactions and affect development. Attach-ment sites may be a limited resource inducing parasite competition. In this respect, monogenean abundance has been correlated to host size, where an increase in fish body length correlates to an increase in gill surface area (Rohde et al. 1995, Barse 1998). In contrast, no mono-geneans were found in fish fry with poorly developed gills (Bagge and Valtonen 1999). Discocotyle sagittata trickle/single infection of comparably-sized fish did not affect parasite abundance, suggesting gill surface is not the factor limiting development during mass invasion. Oncomiracidia and juvenile worms are small relative to gill surface area, and thus should be able to occur con-currently without major space limitations. Our results suggest competitive interactions; however, the experi-mental design compared the relative effectiveness of trickle/single infection and does not offer insight into the mechanisms underlying these putative interactions.

\section{Parasite distribution on the gills}

Adult monogenean parasites have been shown to exhibit preferences for attaching to certain gill arches (Llewellyn 1956). Adult D. sagittata has been reported to occur preferentially on gill arches I and II in both its natural host in Europe, the brown trout Salmo trutta (Llewellyn and Owen 1960, Paling 1965, Gannicott 1997), and an introduced species, the rainbow trout Oncorhynchus mykiss (Gannicott 1997). Paling (1969) suggested $D$. sagittata actively avoided gill arches that are exposed to the greatest water flows (arches II and III), but Gannicott (1997) reported that at high worm burdens $(>50 /$ fish), parasites were found in significantly larger numbers on arch II. In this study, older and sexually mature worms from naturally-infected fish exhibited a preference for the first two gill arches (Table 3), while juveniles tended to occur more frequently on arches II and III (Fig. 6). This would indicate that in the early stages of experimental infection of rainbow trout, parasite distribution on the gills can be explained in terms of the relative amounts of water flowing over each gill arch, as proposed by Llewellyn (1956). The fact that juvenile and adult parasite distributions differ prompts two hypotheses: a) differential mortality: juveniles primarily invade wellventilated areas of the gills which are less favourable and thus experience higher mortality than parasites in less-irrigated areas; b) migration: older worms migrate from the site of initial attachment to the most favourable regions on the gill apparatus. Our results support the second hypothesis, since the numbers

of worms recovered 1, 2 and 3 months p.i. (Table 3 ) are not significantly different, but their distribution is.

We propose that $D$. sagittata oncomiracidia primarily attach to the most ventilated parts of the gills because they are carried by the respiratory current during invasion, and stay in these sites until they have grown 2-3 pairs of clamps, when they start migrating to lessventilated regions on the gills. Adult $D$. sagittata worms have been observed attaching simultaneously to two adjacent gill arches (Gannicott 1997), and freshly attached oncomiracidia have been described to travel by "looping" movements between gill filaments (Gannicott and Tinsley 1998a). Larvae and juvenile worms secure attachment to the host with their clamps, as well as marginal hooks (Llewellyn and Owen 1960, Owen 1970) and adhesive secretions putatively secreted through gland-like structures located anteriorly (Owen 1970, Whittington and Cribb 2000). Migration could proceed via the distal end of filaments, where lamellae from neighbouring arches actually touch each other; this would probably expose parasites to the strongest currents that occur over the whole respiratory apparatus. Alternatively, they could move proximally along the arches themselves into the buccal cavity, translocate to the preferred arch and migrate distally to the lamellae; occasionally, juveniles were observed on the gill rakers. Adult parasites could move from one gill arch to the contiguous one because on fully-grown haptors the distance between the rows of clamps on each extreme would be sufficient to bridge the gap between adjacent gill arches (Llewellyn 1956). The well-developed nervous system in D. sagittata (Cable et al. 1996) would coordinate this manoeuvre.

Migration of monogenean larvae is a well documented phenomenon, especially in the case of Monopisthocotylea (Euzet and Combes 1998); e.g., Entobdella soleae (Kearn 1984), Anoplodiscus cirrusspiralis (West and Roubal 1998) and Neoheterobothrium hirame (Anshary and Ogawa 2001). Adult polyopisthocotylean parasites may migrate to form mating rendez-vous, as has been described for gill parasites of the humbug Dascyllus arunus (Lo 1999). Apart from mechanical differences in attachment capabilities, niche specialisa-tion could arise from distinct metabolic and environ-mental requirements, as well as interspecific competition (Šimková et al. 2000). Monogenean migration has also been shown to be influenced by immune functions, as in the case of Gyrodactylus derjavini infecting rainbow trout (Buchmann and Bresciani 1998, 1999). The present work suggests that $D$. sagittata oncomiracidia invade the gills passively and then migrate to preferential 
attachment sites, and that trickle and single infection are equally successful under controlled laboratory conditions.
Acknowledgements. MRG is supported by a postgraduate scholarship from the Mexican National Council for Science and Technology (CONACYT) and the Overseas Research Student Award Scheme (Committee of Vice-Chancellors and Principals, UK). We thank John Ballard and Ken Jervis for access to fish and for assistance during fieldwork in the Isle of Man.

\section{REFERENCES}

ANSHARY H., OGAWA K. 2001: Microhabitats and mode of attachment of Neoheterobothrium hirame, a monogenean parasite of Japanese flounder. Fish Pathol. 36: $21-26$.

BAGGE A.M., VALTONEN E.T. 1999: Development of monogenean communities on the gills of roach fry (Rutilus rutilus). Parasitology 118: 479-487.

BARGER I.A., LE JAMBRE L.F., GEORGI J.R., DAVIES H.I. 1985: Regulation of Haemonchus contortus populations in sheep exposed to continuous infection. Int. J. Parasitol. 15: 529-533.

BARNES E.H. 1997: Population dynamics of the parasitic stages of Oesophagostomum dentatum in pigs in single and trickle infections. Int. J. Parasitol. 27: 1595-1604.

BARSE A.M. 1998: Gill parasites of mummichogs, Fundulus heteroclitus (Teleostei: Cyprinodontidae): effects of season, locality, and host sex and size. J. Parasitol. 84: 236244.

BOSSAERT K., FARNIR F., LECLIPTEUX T., PROTZ M., LONNEUX J.F., LOSSON B. 2000a: Humoral immune response in calves to single-dose, trickle and challenge infections with Fasciola hepatica. Vet. Parasitol. 87: 103123.

BOSSAERT K., JACQUINET E., SAUNDERS J., FARNIR F., LOSSON B. 2000b: Cell-mediated immune response in calves to single-dose, trickle, and challenge infections with Fasciola hepatica. Vet. Parasitol. 88: 17-34.

BUCHMANN K., BRESCIANI J. 1998: Microenvironment of Gyrodactylus derjavini on rainbow trout Oncorhynchus mykiss: association between mucous cell density in skin and site selection. Parasitol. Res. 84: 17-24.

BUCHMANN K., BRESCIANI J. 1999: Rainbow trout leucocyte activity: influence on the ectoparasitic monogenean Gyrodactylus derjavini. Dis. Aquat. Org. 35: 13-22.

CABLE J., MARKS N.J., HALTON D.W., SHAW C., JOHNSTON C.F., TINSLEY R.C., GANNICOTT A.M. 1996: Cholinergic, serotoninergic and peptidergic components of the nervous system of Discocotyle sagittata (Monogenea: Polyopisthocotylea). Int. J. Parasitol. 26: 1357-1367.

DOBSON R.J., WALLER P.J., DONALD A.D. 1990: Population dynamics of Trichostrongylus colubriformis in sheep: the effect of infection rate on the establishment of infective larvae and parasite fecundity. Int. J. Parasitol. 20: $347-352$.

EUZET L., COMBES C. 1998: The selection of habitats among the Monogenea. Int. J. Parasitol. 28: 1645-1652.

FALL E.H.M., KUMAR V., GEERTS S., SALVOLDI M. 1996: Comparison of single and trickle infections of pigs with eggs of the Asian Taenia. Vet. Parasitol. 61: 231237.

GANNICOTT A.M. 1997: The biology of Discocotyle sagittata (Monogenea) infecting trout. PhD Thesis, University of Bristol, 312 pp.

GANNICOTT A.M., TINSLEY R.C. 1997: Egg hatching in the monogenean gill parasite Discocotyle sagittata from the rainbow trout (Oncorhynchus mykiss). Parasitology 114: 569-579.

GANNICOTT A.M., TINSLEY R.C. 1998a: Larval survival characteristics and behaviour of the gill monogenean Discocotyle sagittata. Parasitology 117: 491-498.

GANNICOTT A.M., TINSLEY R.C. 1998b: Environmental effects on transmission of Discocotyle sagittata (Monogenea): egg production and development. Parasitology 117: 499-504.

GRUNER L., MANDONNET N., BOUIX J., KHANG J.V.T., CABARET J., HOSTE H., KERBOEUF D., BARNOUIN J. 1994: Worm population characteristics and pathological changes in lambs after a single or trickle infection with Teladorsagia circumcincta. Int. J. Parasitol. 24: 347-356.

KEARN G.C. 1984: The migration of the monogenean Entobdella soleae on the surface of its host, Solea solea. Int. J. Parasitol. 14: 63-69.

LLEWELLYN J. 1956: The host-specificity, micro-ecology, adhesive attitudes, and comparative morphology of some trematode gill parasites. J. Mar. Biol. Assoc. 35: 113-127.

LLEWELLYN J., OWEN I.L. 1960: The attachment of the monogenean Discocotyle sagittata Leuckart to the gills of Salmo trutta L. Parasitology 50: 51-59.

LO C.M. 1999: Mating rendezvous in monogenean gill parasites of the humbug Dascyllus arunus (Pisces: Pomacentridae). J. Parasitol. 85: 1178-1180.

McCLURE S.J., EMERY D.L., BENDIXSEN T., DAVEY R.J. 1998: Attempts to generate immunity against Trichostrongylus colubriformis and Haemonchus contortus in young lambs by vaccination with viable parasites. Int. J. Parasitol. 28: 739-746.

OWEN I.L. 1970: The oncomiracidium of the monogenean Discocotyle sagittata. Parasitology 61: 279-292.

PALING J.E. 1965: The population dynamics of the monogenean gill parasite Discocotyle sagittata Leuckart on Windermere trout, Salmo trutta L. Parasitology 55: 667694.

PALING J.E. 1968: A method of estimating the relative volumes of water flowing over the different gills of a freshwater fish. J. Exp. Biol. 48: 533-544.

PALING J.E. 1969: The manner of infection of trout gills by the monogenean parasite Discocotyle sagittata. J. Zool. 159: 293-309. 
ROBERTS R.J. 1978: Fish Pathology. Baillière Tindall, Cassell, New York, 318 pp.

ROHDE K., HAYWARD C., HEAP M. 1995: Aspects of the ecology of metazoan ectoparasites of marine fishes. Int. J. Parasitol. 25: 945-970.

ŠIMKOVÁ A., DESDEVISES Y., GELNAR M., MORAND S. 2000: Co-existence of nine gill ectoparasites (Dactylogyrus: Monogenea) parasitising the roach (Rutilus rutilus L.): history and present ecology. Int. J. Parasitol. 30: 1077-1088.

TALVIK H., CHRISTENSEN C.M., NANSEN P. 1999: Development and infectivity of eggs and larvae derived from pigs trickle-infected with Oesophagostomum dentatum at different dose levels. Parasitol. Res. 85: 83-87.

TATNER M.F. 1996: Natural changes in the immune system of fish. In: G. Iwama and T. Nakanishi (Eds.), The Fish Immune System: Organism, Pathogen and Environment. Academic Press, London, pp. 255-287.

THONEY D.A., HARGIS W.H.J. 1991: Monogenea (Platyhelminthes) as hazards for fish in confinement. Annu. Rev. Fish Dis. 1: 133-153.
VALTONEN E., PROST M., RAHKONEN R. 1990: Seasonality of two gill monogeneans from two freshwater fish from an oligotrophic lake in northern east Finland. Int. J. Parasitol. 20: 101-107.

VAN AKEN D., VERCRUYSSE J., DARGANTES A.P., LAGAPA J.T., RAES S., SHAW D.J. 1997: Pathophysiological aspects of Mecistocirrus digitatus (Nematoda: Trichostrongylidae) infection in calves. Vet. Parasitol. 69: 255-263.

VAN DIEMEN P.M., PLOEGER H.W., NIEUWLAND M.G.B., RIETVELD F.W., PARMENTIER H.K. 1996: Recognition of low molecular weight Cooperia oncophora antigens after primary and trickle infection of calves with third-stage infective larvae. Int. J. Parasitol. 26: 13051310.

WEST A.J., ROUBAL F.R. 1998: Experiments on the longevity, fecundity and migration of Anoplodiscus cirrusspiralis (Monogenea) on the marine fish Pagrus auratus (Bloch \& Schneider) (Sparidae). J. Fish Dis. 21: 299-303.

WHITTINGTON I.D., CRIBB B.W. 2000: Adhesive secretions in the Platyhelminthes. Adv. Parasitol. 48: 101-224.

Accepted 22 March 2002 
Rubio-Godoy, Tinsley: Discocotyle sagittata trickle and single infection 